\title{
SARS-CoV-2 Transmission and Vaginal Delivery
}

\author{
Rujittika Mungmunpuntipantip $^{1}$ (1) $\cdot$ Viroj Wiwanitkit $^{2}$
}

Received: 12 November 2021 / Accepted: 31 December 2021 / Published online: 11 January 2022

(c) Society for Reproductive Investigation 2022

\section{Dear Editor,}

We would like to share ideas on "Unlikely SARS-CoV-2 transmission during vaginal delivery [1]." Fenizia et al. concluded that "hysteroscopy is not an aerosol-generating procedure and the risk of COVID-19 infection is low; therefore, hysteroscopy should not be ruled out in emergencies [1]." For vaginal transmission of COVID-19, it should not occur. According to a previous report, no virus is shredded into the vagina [2]. Additionally, the virus is rarely detected in blood, and blood contact is not the way that COVID-19 is transmitted. Hence, vaginal delivery should be safe. Nevertheless, standard prevention against respiratory aerosol contamination is still required. During vaginal delivery in the labor room, the chance of contamination of the virus in the environment should not be forgotten. If a mother is infected by SARS-CoV-2, a barrier should be applied to prevent direct contact with the newborn and others in the labor room.

\section{Declarations}

Conflict of Interest The authors declare no competing interests.

\section{References}

1. Fenizia C, Saulle I, Di Giminiani M, Vanetti C, Trabattoni D, Parisi F, Biasin M, Savasi V. Unlikely SARS-CoV-2 transmission during vaginal delivery. Reprod Sci. 2021;28(10):2939-41.

2. Takmaz O, Kaya E, Erdi B, Unsal G, Sharifli P, Agaoglu NB, Ozbasli E, Gencer S, Gungor M. Severe acute respiratory syndrome coronavirus (SARS-CoV-2) is not detected in the vagina: a prospective study. PLoS One. 2021;16(9):e0253072.

Rujittika Mungmunpuntipantip

Bangkok, Thailand

Ajeenkya DY Patil University, Pune, India 